\title{
Top polarization, forward-backward asymmetry and new physics
}

\author{
Debajyoti Choudhury ${ }^{a}$, Rohini M. Godbole 1 , Saurabh D. Rindani ${ }^{c}$, Pratishruti Saha ${ }^{a}$ \\ ${ }^{a}$ Department of Physics and Astrophysics, University of Delhi, Delhi 110 007, India. \\ ${ }^{b}$ Theory Unit, CERN, CH-1211, Geneva 23, Switzerland. \\ ${ }^{c}$ Theoretical Physics Division, Physical Research Laboratory, Navrangpura, Ahmedabad 380 009, India.
}

\begin{abstract}
We consider how the measurement of top polarization at the Tevatron can be used to characterise and discriminate among different new physics models that have been suggested to explain the anomalous top forward-backward asymmetry reported at the Tevatron. This has the advantage of catching the essence of the parity violating effect characteristic to the different suggested new physics models. Other observables constructed from these asymmetries are shown to be useful in discriminating between the models, even after taking into account the statistical errors. Finally, we discuss some signals at the $7 \mathrm{TeV}$ LHC.
\end{abstract}

PACS Nos:14.65. Ha, 13.88.+e

Key Words:top, asymmetry, polarization

\footnotetext{
${ }^{1}$ Permanent Address: Centre for High Energy Physics, Indian Institute of Science, Bangalore 560012 , India
} 


\section{Introduction}

The study of the third generation quarks in the Standard Model(SM) fermions continues to throw up surprises. Be it a $\sim 3 \sigma$ deviation in $A_{F B}^{b}$ in the SM fit to the electroweak(EW) precision measurements at LEP [1, a forward-backward asymmetry in top-pair production [2 6] significantly larger than what the SM predicts or the discrepancy in the value of the $B_{s}^{0}-\overline{B_{s}^{0}}$ mixing as suggested by the recent measurement of the inclusive dimuon asymmetry [7] at the Tevatron, some deviations from the SM remain. While, individually, none of them are large enough to merit the status of a discovery of physics beyond the SM, they are, nonetheless, intriguingly poised to warrant serious attention. This is especially so on account of the largeness of the third generation fermion masses. With the top quark mass scale being very close to the EW scale, it is quite conceivable that the third generation (or, at least, the top quark) plays an important role in electroweak symmetry breaking itself. Indeed, very many ideas going beyond the SM quite often also predict discernible new physics effects for processes involving the third generation. On the experimental front, constraints on the universality of interactions are far less restrictive for these fermions as opposed to those for the first two generations. Hence, on very generic grounds, top quark physics studies at the Tevatron and the LHC, hold potential for probing new physics [8]. It is, therefore, a small wonder that very many "explanations" of the physics responsible for the aforementioned discrepancies have been offered and a majority of them accord a special role to the third generation. In this article, we concentrate on the anomaly in the forward-backward (FB) asymmetry in top-pair production.

Within the SM, the dominant $t \bar{t}$ production mode at the Tevatron is a pure QCD one and is FB symmetric at the tree-level. Weak interactions are highly subdominant and give a very small asymmetry $\left(A_{F B} \ll 1 \%\right)$. It is the interference of the tree-level QCD amplitudes and higher order terms that yields the dominant SM contribution to this asymmetry and results in $A_{F B} \sim 5 \%$ [9, 10]. This has led to several authors [11,12] advocating the measurement of $A_{F B}$ as a tool for probing physics beyond the SM.

The CDF and the D0 experiments at the Tevatron kindled a great deal of interest in top-pair production by reporting a FB asymmetry $\left(A_{F B}\right)$ of $17 \%$ [2] and $12 \%$ [3] respectively. 2 . Many possible New Physics (NP) scenarios have been offered as explanations. In a later update, CDF revised this number to 19.3\%. 4] Although the analysis of more data has reduced the significance of the CDF result to about $1.8 \sigma\left(A_{F B}=15 \%\right)[5]$ and D0 has presented a newer value of $8 \%$ [6], the deviation from the SM is still non-negligible and, coupled with the other longstanding discrepancies in certain third-generation observables, continues to elicit much interest $[13$ 18].

Forward-backward asymmetry, at the Tevatron, is defined as

$$
A_{F B}=\frac{\sigma\left(\cos \theta_{t}>0\right)-\sigma\left(\cos \theta_{t}<0\right)}{\sigma\left(\cos \theta_{t}>0\right)+\sigma\left(\cos \theta_{t}<0\right)}
$$

where $\theta_{t}$ is the angle made by the top quark with the direction of the proton in the lab-frame. Experimentally, the measurement is made in the semi-leptonic channel, where the angle $\left(\theta_{h}\right)$ made by the hadronically decaying top(anti-top) with the proton beam and the charge $Q_{l}$ of

\footnotetext{
${ }^{2}$ The measurements reported by D0 are uncorrected for kinematic acceptance.
} 
the decay lepton from the anti-top(top) are together used to construct the net top current in the direction of the proton beam. $\cos \theta_{t}$ above is thus equivalent to $-Q_{l} \cdot \cos \theta_{h}$ [4]. CP invariance is assumed to hold good.

Naively, a non-zero $A_{F B}$ seems to be an indication of some violation of a discrete symmetry and, indeed, most models that purport to explain this anomaly have invoked a parityviolating interaction for the top-quark. While this assumption certainly holds true for any $s$-channel NP contribution to $q \bar{q} \rightarrow t \bar{t}$, clearly, it is not applicable when $t$ - or $u$-channel contributions are present as well. In other words, the measured $A_{F B}$, in the presence of any NP interactions, may accrue from either explicit parity violation(dynamics) or the effects of $t$-(u)-channel propagators(kinematics) or a combination of both. We believe this issue has not been stressed sufficiently in the literature. The new physics explanations have differing levels of parity violation encoded in the chiral structure of the interactions. As this is reflected in the $A_{F B}$ to varying amounts, it would be interesting to construct a probe thereof. The polarization of a single top offers one such probe. In fact, this observation was first made in Ref. [19] in the context of sfermion exchange contributions to $t \bar{t}$ production in $R$-parity violating MSSM (an analog of this model is one of the candidates for an explantaion of the observed $\left.A_{F B}\right)$.

It is a thankful coincidence that the top quark polarization is also a quantity that is amenable to an experimental measurement due to the large mass of the $t$. Being heavy, the top quark decays before it hadronizes and thus the decay products carry the memory of its spin direction. This correlation between the kinematic distribution of the decay products and the top spin direction, can be used to get information about the latter. In fact, many studies exploring the use of the polarization of the top quark as a probe and discriminator of new physics [20], as a means to sharpen up the signal of new physics [21] and to obtain information on $t \bar{t}$ production mechanism [19,22,23] exist in literature. Different probes of the top polarisation which use the above mentioned correlation have been constructed [23] 25], the angular distribution of the decay leptons providing a particularly robust probe due to its insensitivity to higher order corrections [26] and to possible new physics in the $t b W$ vertex [27 29].

A single-top polarization asymmetry $\left(A_{P}\right)$ can be defined as

$$
A_{P}=\frac{\sigma(+)-\sigma(-)}{\sigma(+)+\sigma(-)},
$$

where + or - denote the helicity of the top quark and the helicities of the $\bar{t}$ are summed over. The SM prediction for this arises due to EW effects and is expected to be small. In this note, we explore the predictions for $A_{P}$ originating from the different new physics explanations of the FB asymmetry. Spin polarization studies are a part of the agenda at the Tevatron as well as the LHC [30]. Both the CDF [31] and the D0 32] experiments at the Tevatron have reported measurements of spin correlation coefficients. In addition, CDF also reports a measurement of $t \bar{t}$ helicity fractions [31. We would like to point out that, while these observables may also be able to distinguish between the different NP scenarios under consideration, they involve measurement of the polarization of the top as well as the anti-top and hence, are experimentally more challenging. This is underscored by the fact that the aforementioned measurements are accompanied by fairly large error bars. On the other hand, measurement of $A_{P}$ requires knowledge of the polarization of only the top and thus provides an advantage in terms of the statistics that may be obtained. 
The rest of the article is organised as follows. In the next section, we discuss the rudiments of the models we use as templates, with particular emphasis on the features that are germane to the issue at hand. This is followed, in section 3 , by a comparative discussion of the structure of some observables and their efficacy in distinguishing certain features. The numerical results, pertaining to the resolving powers of various observables, are presented in section 4 . Finally, we summarise in section [6.

\section{Model Templates}

Rather than probe each and every model that has been proposed, we select some that, to our mind, serve as templates. Broadly speaking, four classes of explanations have been suggested. The first two involve new $t$-channel exchanges in $q \bar{q} \rightarrow t \bar{t}$ while the third involves s-channel contributions to the same. For very large masses of the exchanged bosons, all three reduce to four-fermion contact interactions which constitute the fourth category. We do not discuss the last-mentioned explicitly as it can be approached from each of the other cases in the appropriate limit 3 . The particle exchanged in the $t$-channel could either be a scalar or a vector and we shall discuss an example of each. Scalar exchanges in the $s$-channel do not contribute to $A_{F B}$. On the other hand, $s$-channel vector exchanges are fairly commonplace in new physics scenarios and this constitutes our third template. Finally, we omit tensor exchanges because no such well-motivated model leading to large $A_{F B}$ exists. We now discuss each template in turn.

\subsection{A flavour-nondiagonal $Z^{\prime}$}

While additional $U(1)$ gauge symmetries are well-studied and very often well-motivated, it is easy to see that most common variations would not lead to a substantial $A_{F B}$. In models where the $Z^{\prime}$ appears in the $s$-channel in the $q \bar{q} \rightarrow t \bar{t}$ process, the NP amplitude cannot have a non-zero interference with the QCD contributions. Consequently, obtaining the required $A_{F B}$ requires large $Z^{\prime}$ couplings resulting in too large a correction to $\sigma(t \bar{t})$. On the other hand, a flavour-changing $Z^{\prime}[13]$ would appear in the $t$-channel in, say, $u \bar{u} \rightarrow t \bar{t}$. The corresponding contribution to $A_{F B}$ has two sources, viz. kinematic (due to the $t$-channel propagator) and, possibly a dynamic one as well (if the $Z^{\prime}$ coupling is chiral). Analogous $\bar{t} c Z^{\prime}$ or $\bar{c} u Z^{\prime}$ couplings are disfavoured as these would set up flavour-changing neutral currents. Similarly, consistency with $B$-physics observables is simpler if the $Z^{\prime}$ does not couple to the $b$-quark necessitating that the coupling to the top be right-chiral, thus leaving us with a Lagrangian of the form 13 .

$$
\mathcal{L} \ni g_{X} Z_{\mu}^{\prime} \bar{u} \gamma^{\mu} P_{R} t+\text { h.c. . }
$$

The model also includes a small flavor-diagonal coupling to $u$ quarks in order to avoid experimental constraints from like-sign top production data from the Tevatron [13]. However,

\footnotetext{
${ }^{3}$ In principle, there could be scenarios wherein more than one such NP effect could play a role, albeit to different degrees. Once again we desist from discussing these explicitly as the gross features thereof can be deduced by judiciously combining the templates that we do examine hereafter.
} 
this as well as issues regarding anomaly cancellation are not relevant to the discussion at hand and, hence, we shall ignore them.

\subsection{Diquarks}

Particles carrying a baryon number of $\pm 2 / 3$ occur in many models, most frequently in those concerned with grand unification [33]. While both scalars and vectors are possible, the latter would, typically, have a mass as large as the symmetry breaking scale. The scalars can be light, though, and may couple to a pair of quarks. Indeed, an example of such couplings can be found within the minimal supersymmetric standard model (MSSM) if $R$-parity were not conserved. Once again, the presence of such couplings would generate a non-zero $A_{F B}$, for both kinematic and, if the coupling is chiral, dynamic reasons. The fermion assignments within the SM ensures that the couplings are indeed chiral in nature. Of the various different diquark fields that are possible, clearly the one that couples a $u$ to a $\bar{t}$ can generate the maximal amount of $A_{F B}$ for given mass and coupling strength. In other words, the relevant piece of the Lagrangian is [14]

$$
\mathcal{L} \ni \Phi^{a} \bar{t}^{c} T^{a}\left(y_{S}+y_{P} \gamma_{5}\right) u+\text { h.c. },
$$

where $T_{a}$ denotes the appropriate color-coupling structure. As can be expected, the crosssections for $\Phi^{a}$ transforming as a $\overline{\mathbf{6}}$ of $S U(3)_{C}$ differs from that for a $\mathbf{3}$ only in colour factors. We will not discuss the two situations separately and will restrict to the color triplet diquark 4 . While Ref. [14] admits generic $y_{S}$ and $y_{P}$, it is easy to see that consistency in the $b$-sector motivates the choice of right-chiral couplings, viz, $y_{S}=y_{P}$.

\section{$2.3 \quad$ Axigluons}

Originally motivated as residues of unifiable chiral color models [34], wherein the high energy strong interaction gauge group $S U(3)_{L} \otimes S U(3)_{R}$ is spontaneously broken to the usual $S U(3)_{C}$, the axigluon $A_{\mu}^{a}$ was nothing but the octet gauge boson of the broken symmetry having a purely axial vector coupling - of strength $g_{s}$ - with the SM fermions. This model has been probed, at Tevatron, both in the dijet [35] as well as the $t \bar{t}$ channel [12, 36, 37] and masses $\lesssim 1 \mathrm{TeV}$ have been ruled out. Although such a scenario immediately predicts $A_{F B}$, it has the right sign for this quantity only for small values of $m_{A}$, well below the Tevatron limits [12]. For larger $m_{A}$, the sign of $A_{F B}$ flips.

Motivated by this, Ref. [15 considered a variation with a different embedding of the color group into a $S U(3)_{A} \otimes S U(3)_{B}$. The gluon and axigluon are now admixtures of the $S U(3)_{A}$ and $S U(3)_{B}$ with a non-trivial mixing angle $\theta_{A}$. Anomaly cancellation requires a fourth generation of quarks. The new axigluon is 'flavor non-universal' as its couplings with the $3^{\text {rd }}$ and $4^{\text {th }}$ generation quarks are different from those with quarks of the first two generations, the essential trick being to reverse the sign of the axial coupling of the top-quark, thereby

\footnotetext{
${ }^{4}$ Ref. [14] also investigated scalars (with vanishing baryon numbers) in $\mathbf{8}$ and $\mathbf{1}$ representations of $S U(3)_{C}$ but those were not found to be very successful in consistently reproducing the relevant experimental data.
} 
reversing the sign of $A_{F B}$. In the bargain, additional vector-like couplings are introduced for the axigluon, leading to

$$
\mathcal{L} \ni \bar{\psi} \gamma_{\mu}\left(g_{V}^{x}+g_{A}^{x} \gamma_{5}\right) T_{a} \psi A_{a}^{\mu}
$$

While the vector coupling is generation universal $\left(g_{V}^{x}=-g_{s} \cot 2 \theta_{A}\right)$, the axial coupling is not, with $g_{A}^{q}=-g_{s} \operatorname{cosec} 2 \theta_{A}$ for the first two generations and $g_{A}^{t}=+g_{s} \operatorname{cosec} 2 \theta_{A}$ for the last two. Note that, unlike in cases of the diquark and the $Z^{\prime}$, wherein the new physics contribution only appears in the sub-process $u \bar{u} \rightarrow t \bar{t}$, for the axigluon, all quark flavors are involved.

\section{Analytical Issues}

As we are interested in the polarization asymmetry, we must calculate the cross-sections for different final state helicity combinations. While the details are presented in the Appendix, note that, in each case, the square of the amplitude can be decomposed as

$$
\left|\mathcal{M}\left(\lambda_{t}, \lambda_{\bar{t}}\right)\right|^{2}=\mathcal{A}+\lambda_{t} \lambda_{\bar{t}} \mathcal{B}+\left(\lambda_{t}-\lambda_{\bar{t}}\right) \mathcal{C}
$$

where $\lambda_{t}, \lambda_{\bar{t}}= \pm 1$ are twice the helicities of the top (antitop). For observables relating to a single polarization, such as that we are interested in, the other must be summed over.

It is easy to see that while the total $t \bar{t}$ cross-section and $A_{F B}$ receive contributions from only from $\mathcal{A}$, the polarization asymmetry $A_{P} \sim \mathcal{C} / \mathcal{A}$. It is, thus, worthwhile to examine the dependence of these terms on the coupling constants. As far as the diquark is concerned (see eq. A.4), the terms $\mathcal{A}$ (and $\mathcal{B}$ ) are even in both the (pseudo)-scalar couplings $y_{S}\left(y_{P}\right)$, and thus, the ensuing $A_{F B}$ is insensitive to their $\left(y_{S, P}\right)$ sign (and, hence, to the chirality structure of the theory). The term $\mathcal{C}$ being proportional to the product $y_{S} y_{P}$, the polarization asymmetry picks up the chirality structure unerringly. And while eq A.5, does not reflect this explicitly (owing to the fact that a $V+A$ structure was chosen), the story is similar for the $Z^{\prime}$.

For the axigluon, the situation is bit more complicated. There exist pieces in $\mathcal{A}$ (and $\mathcal{B}$ ), that are odd in the individual axigluon couplings, but being parity odd, most of them do not contribute to $\sigma_{t \bar{t}}$. The only such piece that contributes to $\sigma_{t \bar{t}}$ does so only away from the resonance - with the sign reversing as one crosses it - resulting in a subdominant contribution. Of course, $A_{F B}$ is very sensitive to the relative signs of the couplings in question, which was the original motivation of this model vis-a-vis the flavour-universal axigluon. Once again, the sign of $A_{P}$ would provide additional information about the aforementioned relative signs, thereby serving to establish the actual structure of the embedding of $S U(3)_{C}$ in the larger gauge group.

Finally, note that, for each of the three cases, the piece $\mathcal{C}$ has a different angular dependence, including a parity-odd piece. This leads one to hope that the construction of a rapiditydependent $A_{P}$ would lend additional resolving power. 


\section{Numerical Results}

From the discussions in the preceding section, one expects, in general, that each of the three models would be associated with a different correlation between the three observables $\sigma$, $A_{F B}$ and $A_{P}$. However, since the particular values of these quantities depend sensitively on the parameters of the model (i.e. the boson masses and couplings), it is conceivable that the models could lead to similar values for these observables for some unrelated points in the parameter space. Since such a degeneracy would lead to the models being indistinguishable from each other, at least as far as such observables are concerned, we begin by delineating the situations where this might occur.

To this end, we scan the parameter space for each of these models with the restriction that the couplings be perturbativ 5 . For the $Z^{\prime}$, this implies $g_{X}<2 \pi$. For the diquark theory, (to ensure that there are no relative normalization factors between the couplings in the different models under consideration) the same limit is imposed on $g_{y}=\sqrt{2} y$ where, $y \equiv\left(y_{S}^{2}+y_{P}^{2}\right)^{1 / 2}$. In the case of axigluons, the perturbativity condition is imposed on the couplings $g_{A}$ and $g_{B}$ (associated with $S U(3)_{A}$ and $S U(3)_{B}$, respectively) with both $g_{A}, g_{B}<2 \pi$. This, in turn, translates to $10^{\circ}<\theta_{A}<45^{\circ}[15$.

As far as masses are concerned, the lower limits are model-specific. It is easy to appreciate though that, for very large masses, all the models would, essentially, be equivalent to contact interactions. For very large masses, engendering a large enough $A_{F B}$ would, then, need very large couplings, thereby coming into conflict with the perturbativity requirement discussed above. In effect, only $M_{\text {new }} \lesssim 3000 \mathrm{GeV}$ is relevant. Furthermore, we impose direct limits by taking into account various considerations as detailed below.

In case of diquarks, one could argue for $m_{\Phi}>350 \mathrm{GeV}$, motivated by the limits on squark searches at the Tevatron [1. However, for a scalar decaying primarily into a top (or a bottom) and a jet, these limits do not apply and the corresponding limits are expected to be much weaker. Although such a dedicated study is yet to be reported, preliminary investigations [38] suggest that $m_{\Phi} \gtrsim 200 \mathrm{GeV}$ cannot yet be ruled out, and we shall adopt this.

As for the $Z^{\prime}$ model, much depends on the flavor structure of its coupling. If the $Z^{\prime}$ is very light, then the branching fraction for $t \rightarrow Z^{\prime}+u$ is no longer negligible. Further, if the $Z^{\prime}$ decays hadronically, this would lead to an enhancement in the contribution to the $t \bar{t}$ production from the lepton + jets channel as compared to the contribution from the dilepton channel giving rise to deviations from the experimental measurements in the two channels. Ref. [13] has already considered this to find that $m_{Z^{\prime}} \gtrsim 120 \mathrm{GeV}$ is consistent with current data and we shall adopt this limit as well.

The limits on the flavor non-universal axigluon mass need more thought though. Experimental searches, so far, have only considered flavor universal axigluons. Based on these,

\footnotetext{
${ }^{5}$ We impose this condition only until the $10 \mathrm{TeV}$ scale. Were we to demand that the theory remain perturbative until a significantly higher scale, the parameter space would be further restricted. However, in the absence of any knowledge of the ultraviolet completion, we desist from this.
} 
$m_{A} \lesssim 1250 \mathrm{GeV}$ is ruled out by direct searches while the distortion in the $m_{t t}$ spectrum [39] is too severe for $m_{A} \lesssim 1400 \mathrm{GeV}$. However, it should be noted that the resonant production cross sections for flavor non-universal axigluons differ on account of the differences in their coupling to light quarks. Nonetheless, we do restrict to $m_{A} \gtrsim 1400 \mathrm{GeV}$.

The parameter spaces thus defined, the numerical calculation is done using a parton level Monte Carlo routine. We use the CTEQ6L [40] parton distributions with the factorization scale set to $m_{t}$ with the later held to $172.5 \mathrm{GeV}$ to be consistent with the value used in measurements of cross-section [41] and $A_{F B}$ [5]. A $K$-factor [42] of 1.33 is used to estimate the cross-section at NLO6.

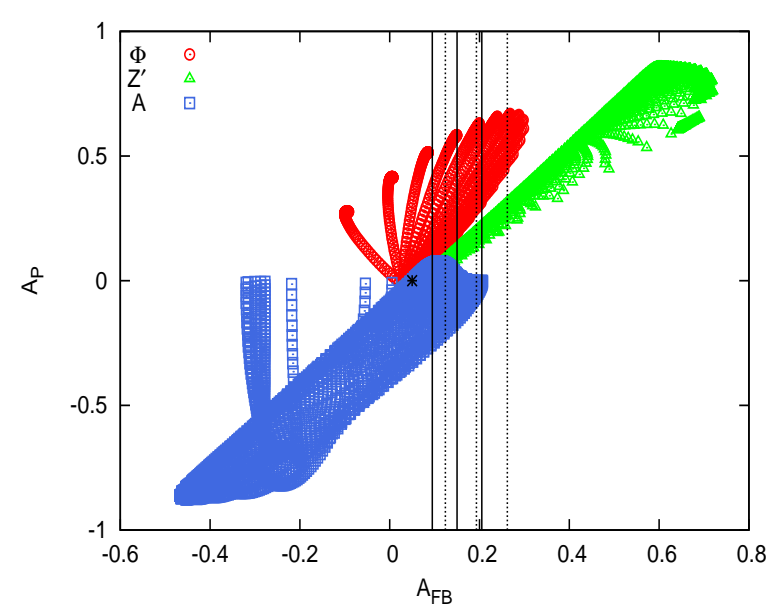

(a)

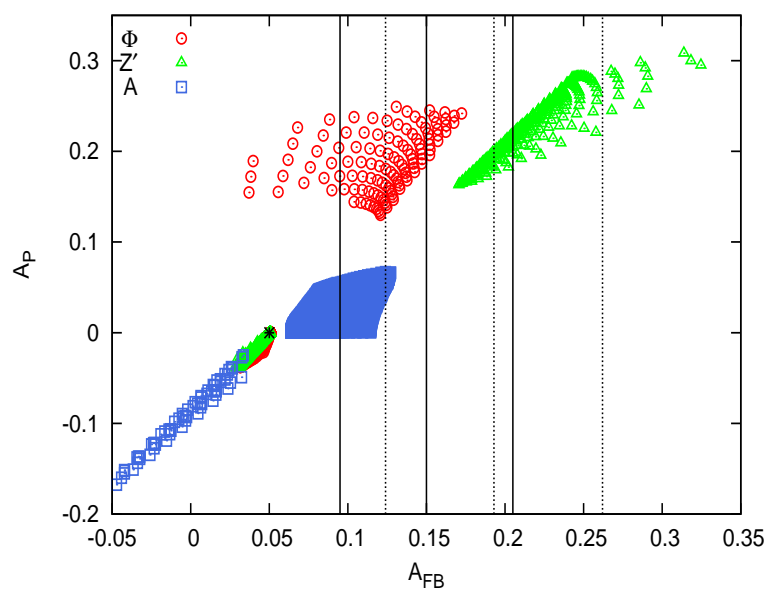

(b)

Figure 1: (a) Correlation between $A_{P}$ and $A_{F B}$ for different models. The vertical solid (dotted) lines correspond to the central value and 1- $\sigma$ bands of the new (old) CDF measurement of $A_{F B}$, namely $15.0 \% \pm 5.5 \%(19.3 \% \pm 6.9 \%)$. The 'star' corresponds to the SM value at NLO. For each model, the scan is limited to only perturbative coupling strengths. (b) As in the previous panel, but restricted to only those points that are consistent with the experimentally observed cross-section at the 1- $\sigma$ level and with restrictions on $M_{\mathrm{boson}}$ as described in the text.

For each parameter-space point we calculate $\sigma_{t \bar{t}}, A_{F B}$ and $A_{P}$ as defined above. Our calculation of $A_{F B}$ includes the SM part [9]. As for $A_{P}$, the SM contribution is miniscule. This is primarily because a non-zero $A_{P}$ can arise only when the production mechanism treats positive and negative helicity states differently. Consequently, a pure QCD process can never give rise to a non-zero $A_{P}$. In the SM, $A_{P}$ will arise only due to electroweak effects, either at the tree level or through mixed EW-QCD higher order corrections, and is, hence, rather small $\left(\mathcal{O}\left(10^{-3}\right)\right)$. Given this, we restrict ourselves to the tree-level value of $A_{P}$ as indicated in the previous section.

In Fig, 1(a), we show the correlation between $A_{P}$ and $A_{F B}$ for each of the three models alongwith the experimentally allowed $1-\sigma$ band for $A_{F B}$. No restrictions have been imposed on the physical observables, barring the aforementioned perturbative limit on the couplings. Note that the splayed-finger like structure for $\Phi$ is but a reflection of the sampling density in the masses and a finer sampling would have resulted in a dense region in this plane. It

\footnotetext{
${ }^{6}$ In the absence of NLO calculations that incorporate the new physics effects under consideration, we use the SM K-Factor.
} 
immediately transpires that the three models under discussion have very different correlations between the observables. However, this is evidently misleading as a large faction of the parameter space depicted here gives rise to a $A_{F B}$ well outside the experimentally allowed range. This is even more so for the total cross section. Clearly, large values of the asymmetries would occur only for substantially large NP couplings and these, typically, give rise to too large a cross section.

Imposing the restriction that the total $t \bar{t}$ production cross section should lie within $1 \sigma$ of the experimentally observed value and restricting $M_{\text {boson }}$ as described earlier, eliminates bulk of the parameter points and leads to the correlation plot of Fig.1(b). One feature immediately stands out. For each of the models, the allowed regions split into two, one close to the SM point and the other distinctly separate.

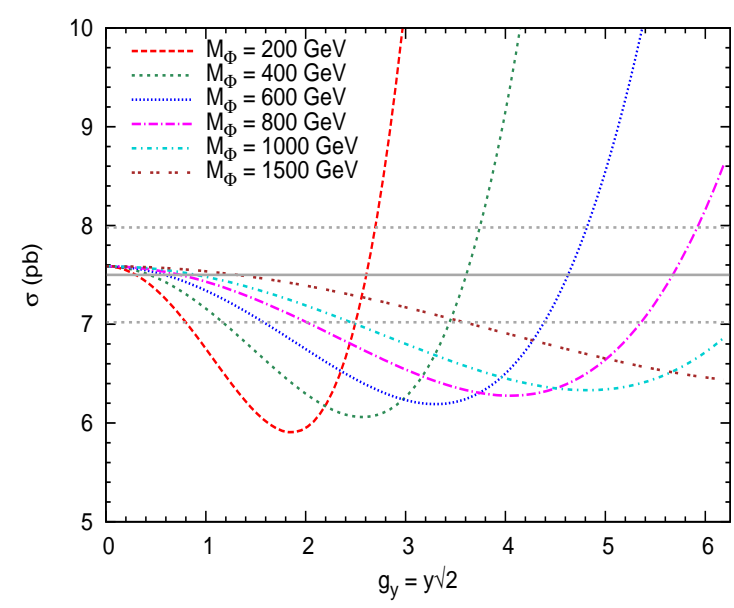

(a)

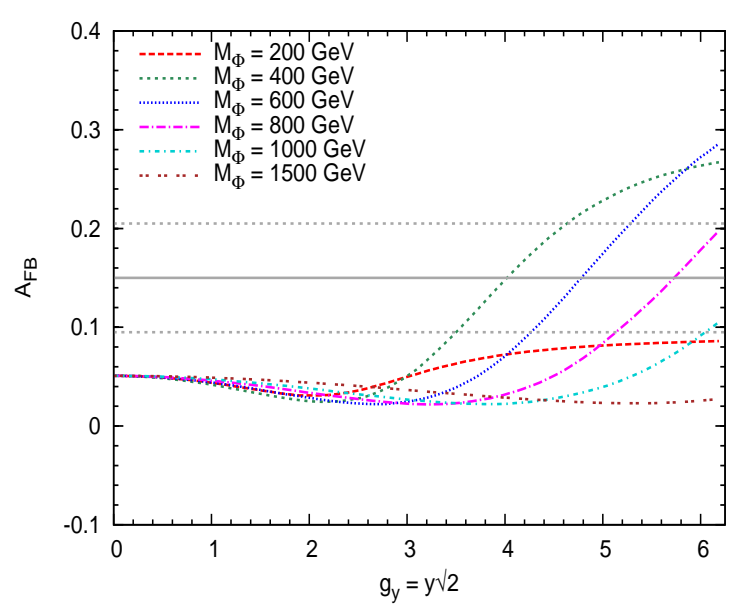

(b)

Figure 2: Variation in $\sigma(t \bar{t})$ and $A_{F B}$ with coupling for different choices of $M_{\Phi}$.

For the $Z^{\prime}$ and the diquark, the reason for this is easy to understand. Clearly, for small values of the NP coupling, the cross-section is close to that within the SM and, hence, consistent with the measured value. However, for such small couplings, the $A_{F B}$ generated is also small and not in consonance with the experimental observation. For a given boson mass, as one considers larger values of the coupling, sufficient $A_{F B}$ can be produced while the cross-section is prevented from becoming too large because of the destructive interference between the SM and NP pieces. In fact, for an intermediate range of the coupling, the cross section falls well below the SM value. The location and the extent of the range naturally depends on the mass of the boson. For the case of the diquark, this is illustrated in Fig, The situation is analogous for the $Z^{\prime}$.

For the axigluon, the situation is somewhat more complicated. Here the interference may be constructive or destructive depending upon the values of $\hat{s}$ and $M_{A}$ under consideration. For $M_{A}>1400 \mathrm{GeV}$, the interference is mostly destructive though. For $\theta_{A} \approx 45^{\circ}$, the situation is identical to that in the case of the flavor universal axigluon, with the exception that now the relative minus sign between the $g_{A}^{q}$ and $g_{A}^{t}$ yields $A_{F B}$ that is of the right sign as well as the right magnitude. As $\theta_{A}$ decreases, the destructive interference takes over leading to $\sigma(t \bar{t})$ values that are too low (see Fig $3(\mathrm{a})$. For $M_{A}<2000 \mathrm{GeV}$, a further increase in the strength of the couplings allows the pure NP term to become dominant and the cross-section increases once again to become consistent with data. Due to this, a part of the range of $\theta_{A}$ 
below $25^{\circ}$ gets ruled out. Again, the actual location and extent of the range depend on the particular value of $M_{A}$. On the other hand, for $\theta_{A} \lesssim 20^{\circ}, A_{F B}$ is only produced in small amounts (Fig, 3(b)]. The resultant picture that emerges is the tail of points near the bottom left of Fig, 1(b) which are consistent with the measurement of $\sigma(t \bar{t})$ but not with that of $A_{F B}$.

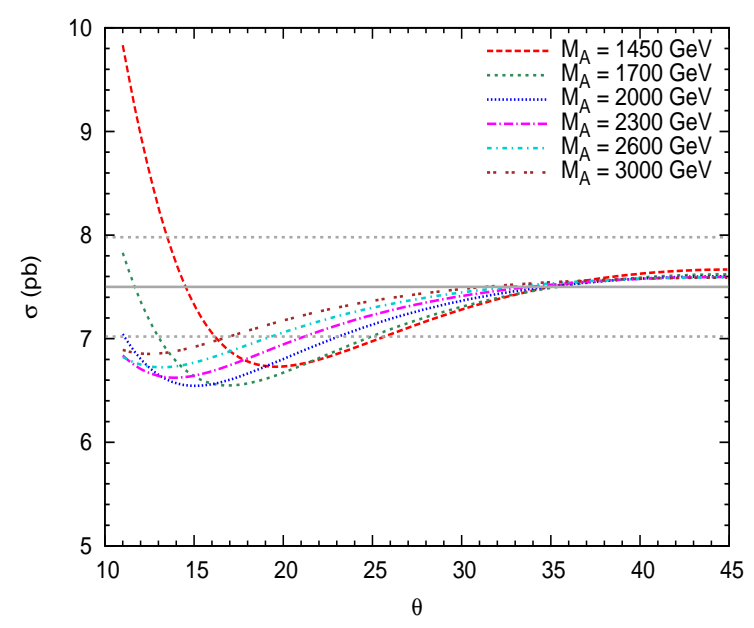

(a)

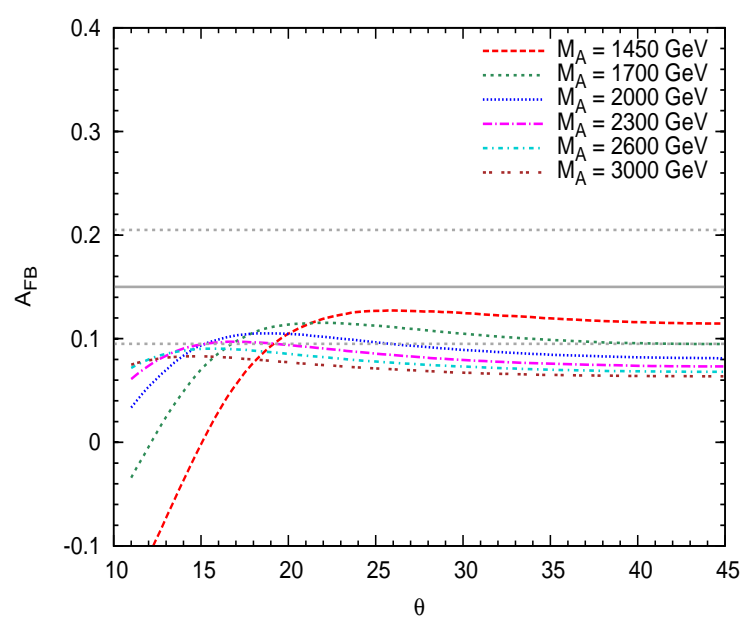

(b)

Figure 3: Variation in cross-section and $A_{F B}$ with $\theta$ for different choices of $M_{A}$.

It is interesting to note that, in Fig $1(\mathrm{~b})$, for the regions that are consistent with the measured $A_{F B}$ values (whether the old measurement or the new one), there is no overlap between the three models. It is true that, consistently flipping the relative sign between the axialand vector-like couplings, 7 , would flip the sign of $A_{P}$ while $\sigma$ and $A_{F B}$ remain unaltered. However, even after such a variation, the flavor non-universal axigluon case would still retain its distance, in the $A_{F B}-A_{P}$ plane, from the other two models. Thus, a measurement of $A_{P}$ should, in principle, be able to distinguish the models even before the corresponding boson has been directly observed, this being particularly true of the axigluon.

Let us also note at this point that analyses of Ref. [23] indicate that polarization values of around 15\%-20\% can be measured at the Tevatron with $10 \mathrm{fb}^{-1}$, with good significance. Further, in addition to the azimuthal asymmetry explored in Ref. [23], a simple forward backward asymmetry of the decay lepton w.r.t. the beam direction, may also be used at the Tevatron and hence one would be able to increase the sensitivity further. From Fig. 1(b) one sees thus that the values of polarization predicted by the different models seem large enough to afford measurement at the Tevatron.

While discrimination of the axigluon model from the other two seems clear, that between the diquark and the Z' models is not so straightforward. For one, they lead to overlapping ranges in both $A_{P}$ and $A_{F B}$. Furthermore, Figs 1 have been drawn with the error bars suppressed. Incorporating the latter would render the two apparently disparate population zones overlapping. If, however, one were to flip the relative sign between the scalar and pseudoscalar couplings of the diquark, the dissimilarity between the models would become quite conspicuous.

\footnotetext{
${ }^{7}$ Of course, this would necessitate a different embedding in the gauge group.
} 


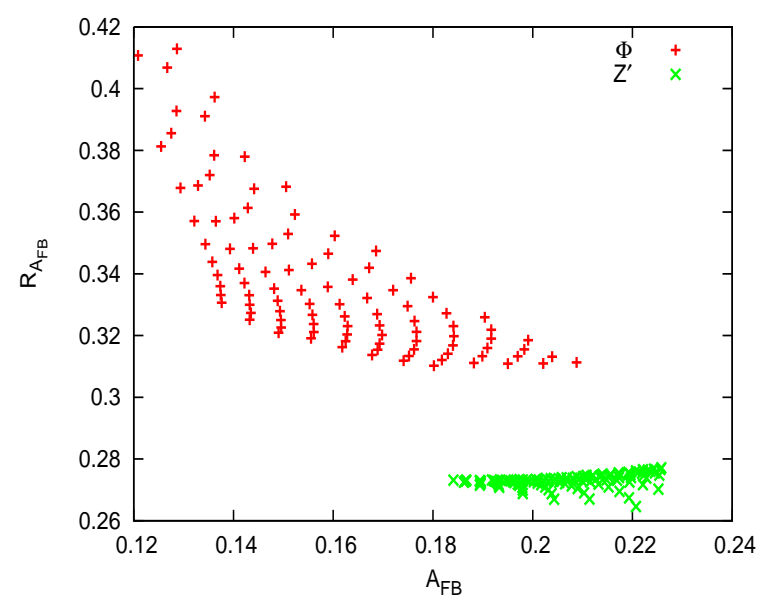

(a)

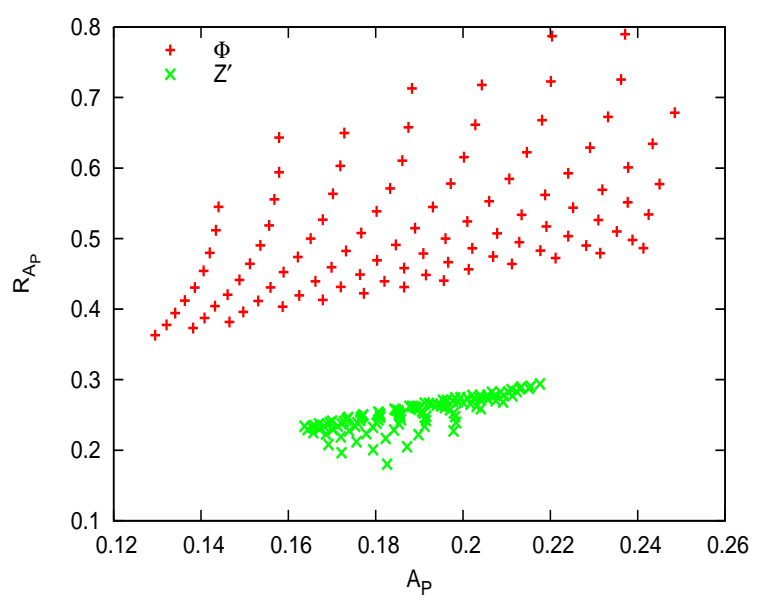

(b)

Figure 4: $R_{A}$ vs $A$ for forward-backward asymmetry and polarization asymmetry. The points depicted are consistent with the CDF measurementc of $\sigma(t \bar{t})$ and $A_{F B}$ at the 1- $\sigma$ level. Also imposed are the perturbativity constraint on the couplings and the constraints on $M_{\mathrm{boson}}$ as described in the text.

Nevertheless, even without changing the relative signs of $y_{S}$ and $y_{P}$ and with a view to making a more robust distinction between the two models, we propose observables that exploit the rapidity distributions in the two scenarios. With the typical values of the $t$-channel masses being different in the two scenarios (see Figs. $2 \& 3$ ), it is but natural that the typical rapidities would be different. To this end, we define the ratios $R_{A_{P}}$ and $R_{A_{F B}}$ where

$$
R_{A}=\frac{A(|\Delta y|<1)}{A(|\Delta y| \geq 1)}
$$

and $\Delta y$ is the difference between the rapidities of the top and the anti-top. The CDF Collaboration does report a measurement of $A_{F B}$ in different $\Delta y$ regions [5], leading to $R_{A_{F B}}=0.043 \pm 0.194$ with the SM expectation being $R_{A_{F B}}(S M)=0.317 \pm 0.053$. While this seems to offer a potentially strong discriminator, as we shall soon see, the associated errors do not, yet, allow for discrimination between models. Instead, we advocate an examination of correlations involving $R_{A_{P}}$ and $R_{A_{F B}}$.

In Fig, 4, $R_{A_{F B}}$ and $R_{A_{P}}$ are plotted against $A_{F B}$ and $A_{P}$ respectively. For consistent comparison with CDF results [5], the asymmetries are calculated in the $t \bar{t}$ rest frame and include the NLO contribution from the SM [5]. It can be seen that the diquark and $Z^{\prime}$ models populate different regions in the $R_{A_{F B}}-A_{F B}$ and $R_{A_{P}}-A_{P}$ planes and the separation is quite distinct.

Once again, Fig. 4 does not incorporate errors in the measurement of the quantities plotted. The statistical part thereof can be easily estimated. We obtain the efficiencies (i.e. including kinematic cuts, event selection efficiencies etc.) from the number of events reported in the CDF $A_{F B}$ measurement [5], and assume that the corresponding efficiencies for $A_{P}$ measurement would be similar. Calculating the expected number of events in this way, we then add the errors in quadrature. While this might seem a trifle optimistic, we compensate by restricting our projections to an integrated luminosity $\left(\sim 4.2 \mathrm{fb}^{-1}\right)$ less than what has already been analysed in Ref. [5]. 


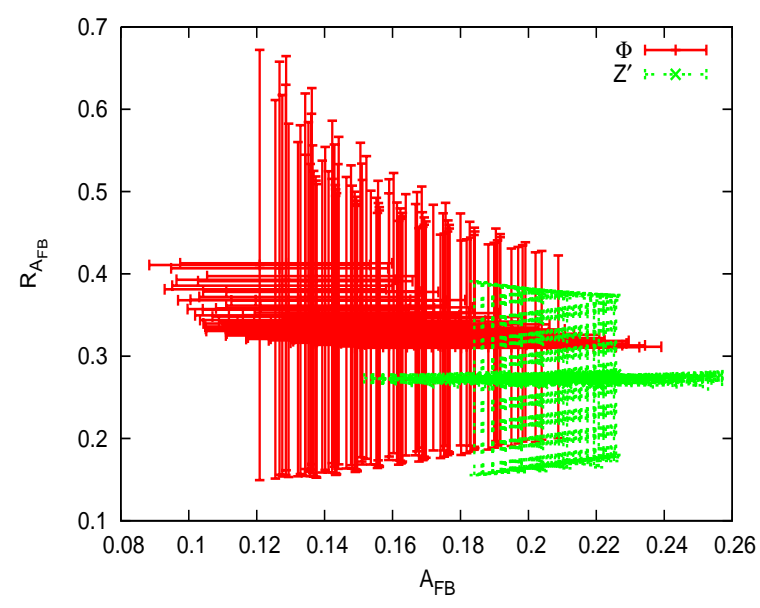

(a)

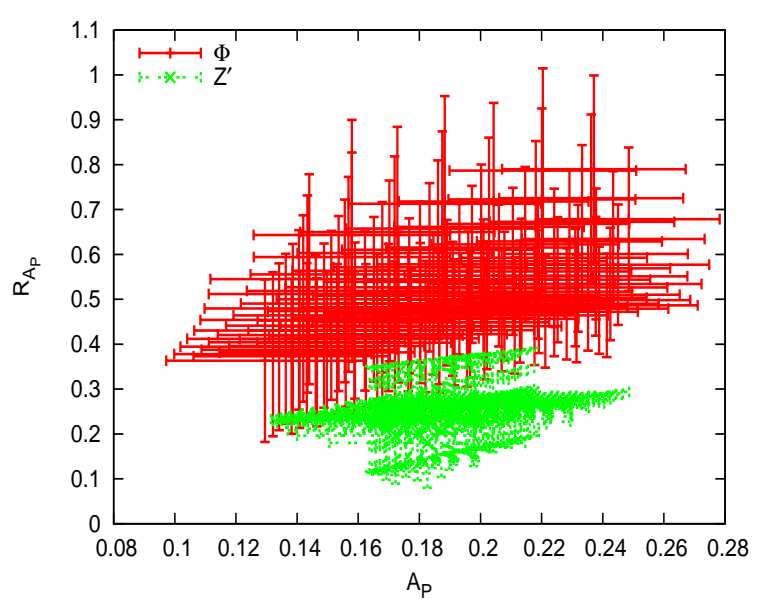

(b)

Figure 5: Correlations involving $A_{F B}, R_{A_{F B}}, A_{P}$ and $R_{A_{P}}$ after inclusion of error bars. The

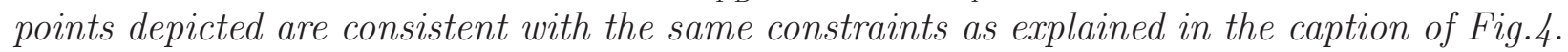

In Fig 5 , we redraw Fig 4 along with the error bars computed as described above. Although the central values in the two scenarios are widely separated, a significant overlap between the error bars persists, thus makes it difficult to distinguish between them. Indeed, the size of the errorbars also shows that the $R_{A_{F B}}$ measurement, on its own, is, as yet, incapable of conclusively distinguishing the models from even the SM. On the other hand, the correlation between $R_{A_{P}}$ and $R_{A_{F B}}$, (Fig, 6) looks more promising. While some overlap exists still, the extent of overlap is distinctly smaller. While a quantitative analysis confirms this, we desist from sharpening our conclusions at this stage. It should also be realized that an analysis more sophisticated than what we have performed and including all the data on tape, is quite likely to further reduce the relative sizes of the errors.

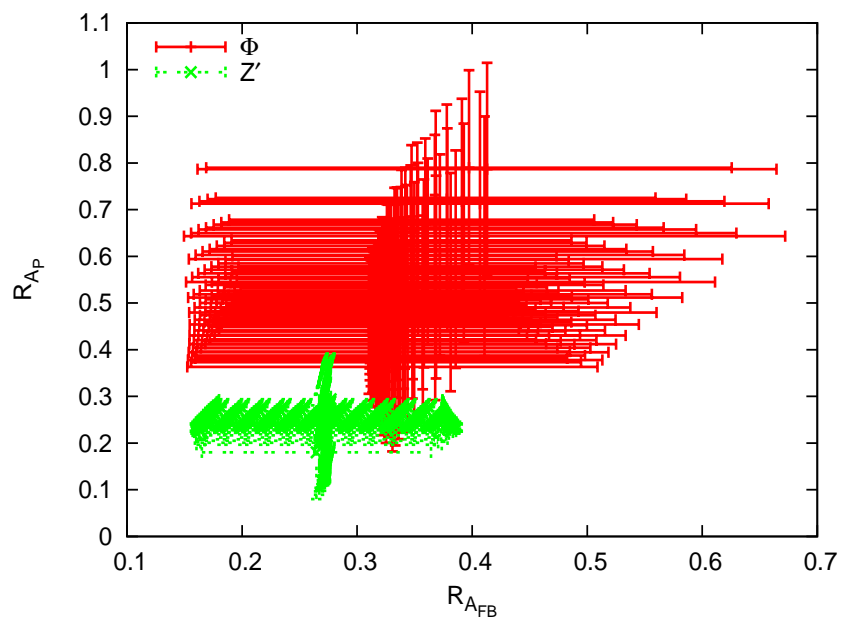

Figure 6: Correlation between $R_{A_{F B}}$ and $R_{A_{P}}$. The points depicted are consistent with the

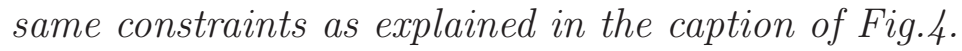

It should be realized that $R_{A_{P}}$ is not the only direction-dependent asymmetry variable. Of the many such possible, we consider only one namely $A_{P}$ as calculated separately for the forward and backward hemispheres. In particular, we plot, in Fig, 7 , the ratio of $A_{P}$ in the two hemispheres against the total $A_{P}$. Again, one finds that the diquark and $Z^{\prime}$ models give rise to markedly different correlations. 


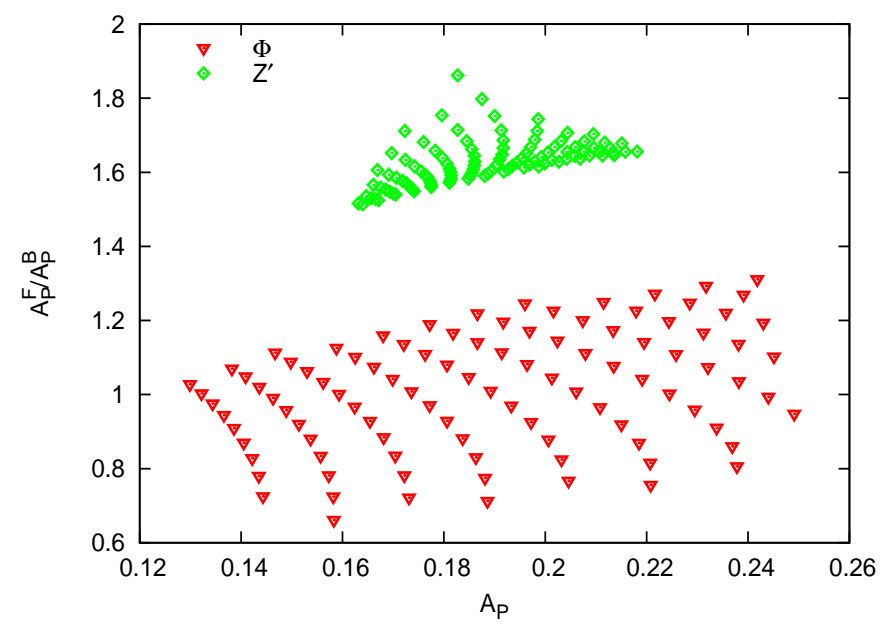

Figure 7: Correlation between the ratio of $A_{P}$ in the forward and backward hemispheres and total $A_{P}$. The points depicted are consistent with the same constraints as explained in the

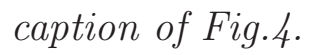

However, as the experience with $R_{A_{P}}$ has shown, the effect of the separation is likely to be diluted by the errors involved. With experimental errors under control, a combination of observables as described above, can be expected to distinguish between the "explanations" for the $A_{F B}$ "anomaly" quite successfully.

\section{Observables at the $\mathrm{LHC}$}

The initial state being symmetric at the LHC implies that no simple forward-backward asymmetry w.r.t the beam direction can be defined. Hence, we limit ourselves to the correlation between $A_{P}$ and $\sigma(t \bar{t})_{8}^{8}$. For the parameter space points that are consistent with the Tevatron measurements of cross-section and $A_{F B}$ at the 1- $\sigma$ level, we find that the three models correspond to different correlations (see Fig. 8). In going from the Tevatron to the LHC, the relative contribution from the $g g \rightarrow t \bar{t}$ sub-process (which does not contribute to such asymmetries) increases as compared to the $q \bar{q} \rightarrow t \bar{t}$ subprocess. The quark initiated subprocess suffers a further suppression as anti-quark fluxes are diminished at a $p p$ collider. As a result, the magnitudes of $A_{P}$ at the LHC are lower than those observable at the Tevatron. Nonetheless, it is interesting to see that the separation of the expected values of $A_{P}$ into different islands is a trend that persists. That the $A_{P}$ for the axigluon is now almost universally negative (as oposed to the Tevatron where positive $A_{P}$ were allowed) is but a consequence of the fact that a resonance is more easily hit at the LHC.

It should also be borne in mind here that the values of polarization presented here correspond to those obtained by integrating over the entire kinematical range. It is clear that by making suitable cuts on the observables such as $m_{t \bar{t}}$ and/or $p_{T}^{t}$, the polarization may be enhanced substantially, as has been seen in Ref. [23. It is also clear that the optimal cuts will be again different for different production mechanisms of the NP top pairs.

\footnotetext{
${ }^{8}$ The K-Factor in this case is estimated by taking the ratio of the NLO cross-section quoted in Ref. 43 . and our own LO calculation resulting in $\mathrm{K}=1.8$.
} 


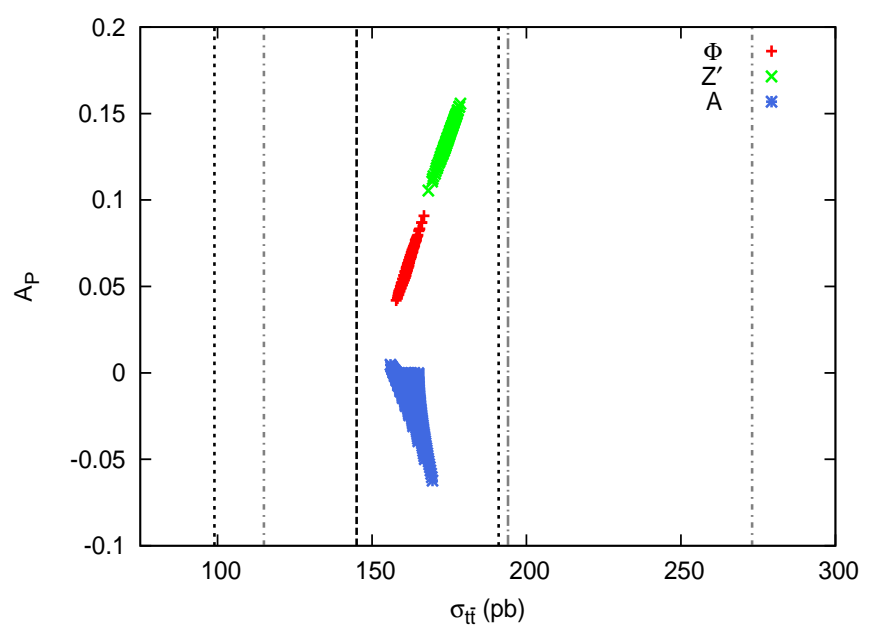

Figure 8: Correlation between $A_{P}$ and $\sigma(t \bar{t})$ at the $L H C(\sqrt{s}=7 \mathrm{TeV})$. The vertical lines show the 1- $\sigma$ interval of the CMS [43] (dot-dashed) and ATLAS [44] (dashed) measurements of the $t \bar{t}$ cross-section. The points depicted are also consistent with the constraints mentioned in the caption of Fig鸟

At the LHC, there also exists the possibility of direct detection of these models through the production of the corresponding boson. For diquarks and axigluons (albeit, the flavor universal variety), the production cross-sections have been calculated in Refs. [14] and [12] respectively. For the $Z^{\prime}$, direct detection is possible through pair production of $Z^{\prime} \mathrm{s}$ or production of a $Z^{\prime}$ in association with a $t$. We present the corresponding cross-sections for these processes in Fig. 9, assuming that eq.(2.1) is the only term in the interaction Lagrangian.
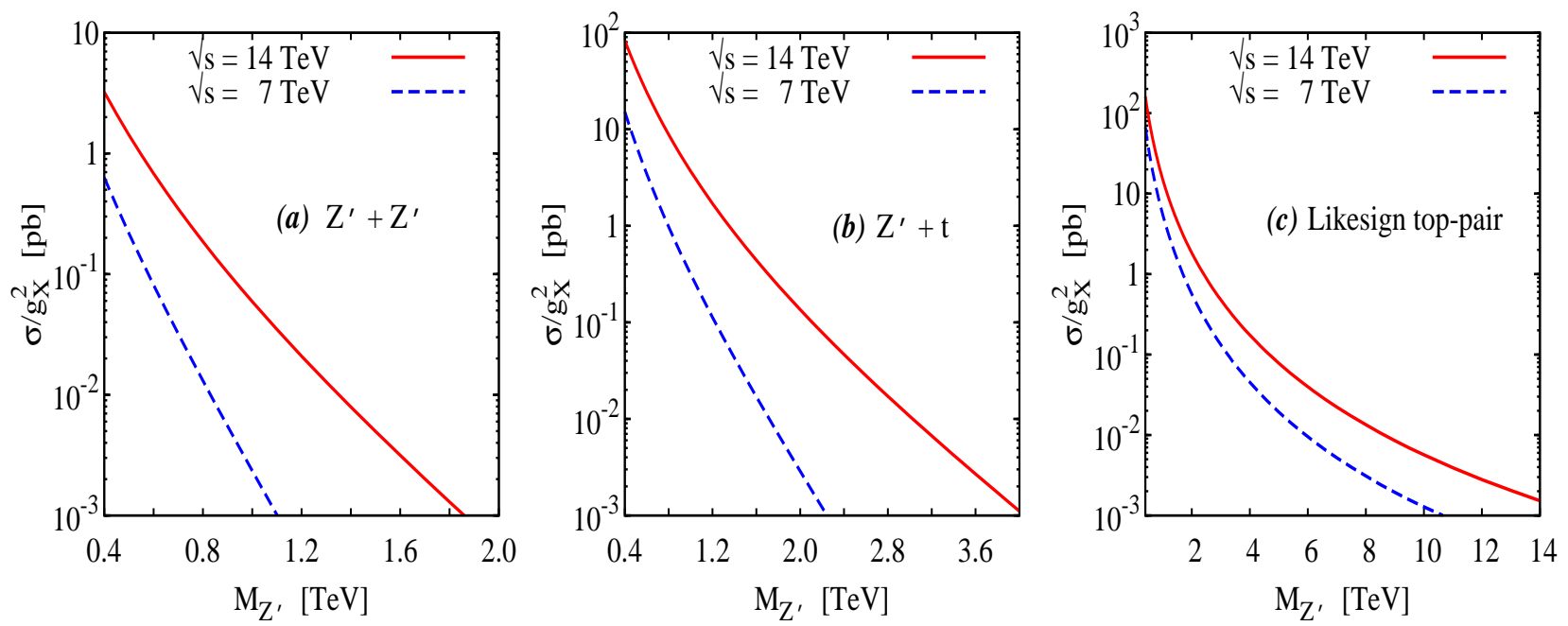

Figure 9: Several production cross sections at the LHC for the $Z^{\prime}$ model. Note that obtaining the requisite $A_{F B}$ at the Tevatron requires the coupling to be typically larger $\left(g_{X} \gtrsim 1\right)$.

Although the cross-sections for the first two processes seem quite large, claims about detectability should be made cautiously. Note that such $Z^{\prime}$ would decay into a top and a light quark (unless other couplings are switched on). Thus, these production processes would, essentially, lead to a $t \bar{t}$ pair accompanied by one or two hard jets. The QCD background for the same is quite large. While invariant mass reconstruction would, in principle, distinguish between signal and background, in practice this is not an easy task [38], especially with two top-quarks present. This observations holds for diquark production as well. 
Relatively easier to look for is the production of a like-sign top-pair, for which this model predicts a large rate. Indeed, as Fig. 9(c) shows, with the projected luminosities at the LHC, such a $Z^{\prime}$ could be measured upto several TeVs even for $g_{X}=1$ (which, essentially, takes us to the limit of a contact interaction). It should be noted, though, that a very large $m_{Z^{\prime}}$ necessitates a large $g_{X}$ for producing the correct $A_{F B}$. Such a scenario should have made its presence felt in the very same process at the Tevatron itself.

\section{Summary}

The anomalously large forward-backward asmymmetry in top-pair production observed at the Tevatron continues to puzzle. Several models have been proposed to "explain" this. While some of them have their roots in well-motivated scenarios originally proposed to address other issues, some of the others are purely phenomenological in nature. The very fact that many different models of new physics can explain this anomaly renders difficult the identification of the best solution.

In this note we have analysed the role that the longitudinal top polarization $\left(A_{P}\right)$ can play in discriminating between such models. The scenarios proposed, typically, differ from each other in their chiral structure and in the relative amount of FB asymmetry that is generated by the dynamics as opposed to kinematic effects. $A_{P}$, being a pure parity violating effect, probes the former aspect.

To illustrate this point we choose from among the different proposed models, three templates and calculate the predictions for $A_{P}$ in each. As expected, we find different correlations between $A_{P}$ and $A_{F B}$ in the three cases and some scenarios can be clearly distinguished from others purely on this account. However, bearing in mind that experimental errors may reduce this apparent separation between the models, we further construct the ratios $R_{A} \equiv A(|\Delta y|<1) / A(|\Delta y|>1)$, with $\Delta y$ being the rapidity separation between the top pair. Correlation between $R_{A_{P}}-R_{A_{F B}}$ seems quite promising in its ability to separate the different models even after the inclusion of errors. Given that such measurements are already being made at the Tevatron, we hope that the adoption of our algorithm would serve to solve this vexing issue.

We also look at the case of the LHC briefly. Again, a scan over the parameter ranges of the three template models, consistent with the Tevatron data, shows that they occupy three disjoint islands in the $A_{P}-\sigma_{t \bar{t}}$ space. Although the magnitude of $A_{P}$ is smaller, owing to the increased importance of the $g g \rightarrow t \bar{t}$ subprocess, the situation could be improved further by the adoption of apropriate kinematic restrictions. In this particular exploratory exercise, as far as the LHC is concerned, we refrain from carrying out such studies.

While most of the models to explain $A_{F B}$ had been chosen by the respective authors, so as to evade current direct search bounds, this would not be the state of the matter in the LHC era. As we have demonstrated, the production cross-sections for new particles inherent to such models, are likely to be large at the LHC. Given the peculiar couplings of such particles, commensurate search strategies need to be devised, but, by no means, 
is this an insurmountable problem. Indeed, even if the new particles were all heavy, the effective Lagrangian responsible for generating the $A_{F B}$ would, typically, leave its mark on SM processes at the LHC (see Sec 5 ).

It should be noted that, by their very nature, most of the scenarios proposed are phenomenogical in nature and have concentrated on explaining $A_{F B}$ while taking care of consistency with only the most obvious of other observables. Indeed, a global fit would render many of them inconsistent. For example, the diquark model would take $R_{\ell} \equiv \Gamma(Z \rightarrow$ hadrons $) / \Gamma\left(Z \rightarrow \ell^{-} \ell^{+}\right)$ away from the LEP/SLC measurements [45. As for the $Z^{\prime}$ model, the couplings and masses required to give a good fit to both $\sigma_{t \bar{t}}$ and the new measurements of $A_{F B}$ are disfavoured by the lack of observation of like-sign top pairs at the Tevatron [13]. The flavor non-universal axigluon, on the other hand, is disfavoured by $B$-physics observables [17]. While such problems might seem specific to these models, variations of these and others such are almost endemic to all such endeavours. They can, of course, be cured by introducing compensatory effects in the respective models. Thus, rather than rejecting them on account of such disagreements, direct searches are the best bet.

It is here that algorithms such as those we propose are particularly useful. Apart from discriminating betweeen models, such analyses can also serve to form an information basis on which more sophisticated model-building can be based.

Note Added : As we were finalising the manuscript, two papers, [46] and [47], have appeared. In the former, the authors have studied different polarization observables, one of them being the polarization of the $t$ quark that we consider. While they look at different models proposed to explain the $A_{F B}$, they concentrate solely on the LHC (at $7 \mathrm{TeV}$ as well as $14 \mathrm{TeV}$ ) and do not consider correlation with $A_{F B}$. Ref. [47], on the other hand, looks at the effects at the Tevatron but it is an extension of model-independent analysis of $A_{F B}$ done in their earlier paper [18] to predictions for correlations of $t$ and $\bar{t}$ polarizations. Since CP conservation relates $t$ and $\bar{t}$ polarizations, we consider it sufficient to study the polarization of either one of $t$ or $\bar{t}$, which gives us the advantage of larger statistics. Further, the analysis of Ref. [18] is valid only when masses of exchanged particles are much higher than the cut off scale and hence there is no direct, easy comparison between the two approaches.

After the submission of our manuscript, the CDF Collaboration published a new analysis of $A_{F B}$ [48] including its dependence on the mass and rapidity of the $t \bar{t}$ pair. The analysis in different rapidity regions is identical to that in Ref. [5] which we have already considered. Further, Ref. [48] reported that $A_{F B}$ is found to be larger in the high invariant mass region $\left(m_{t \bar{t}} \geq 450 \mathrm{GeV}\right)$ than in the low invariant mass region $\left(m_{t \bar{t}}<450 \mathrm{GeV}\right)$. While Ref. [48] itself pointed out that the analysis is still fraught with theoretical uncertainties, we carried out a preliminary investigation which showed that this feature is borne out by all the model templates that we have studied (Fig 10) and does not offer any additional resolution between the models under consideration. In fact, this was to be expected in view of the facts that $(a)$ the total cross section, which is dominated by the small $m_{t \bar{t}}$ range agrees very well with the SM expectations and $(b)$ the measured $A_{F B}$ is substantial. Furthermore, even if uncertainties, both theoretical and experimental, can be reduced, the crucial fact is that, unlike $A_{P}$, this observable has little sensitivity to the chiral nature of the new physics couplings. 


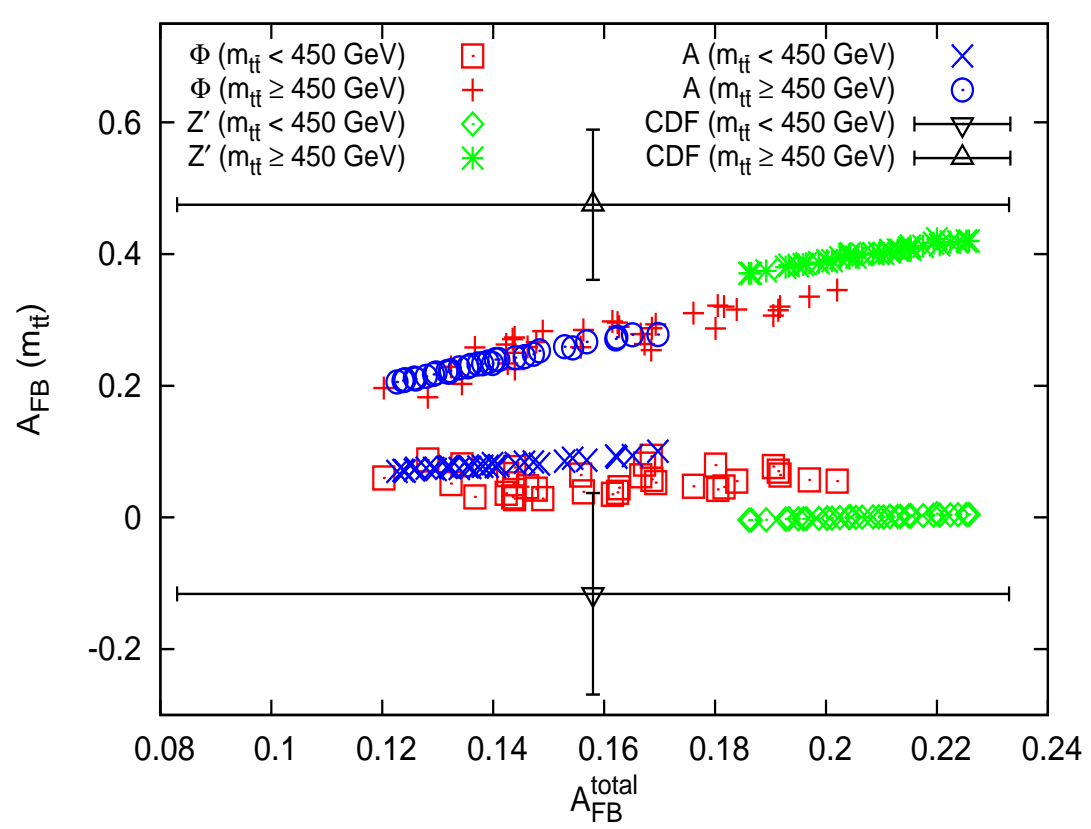

Figure 10: $A_{F B}$ in the regions $m_{t \bar{t}}<450 \mathrm{GeV}$ and $m_{t \bar{t}} \geq 450 \mathrm{GeV}$ compared to the total $A_{F B} . A_{F B}$ is calculated in the $t \bar{t}$ rest frame. The points depicted are consistent with the same constraints as explained in the caption of Fig囵

\section{ACKNOWLEDGEMENT}

R.G. and S.D.R. wish to acknowledge support from the Department of Science and Technology, India under the J.C. Bose Fellowship scheme under grant nos. SR/S2/JCB-64/2007 and SR/S2/JCB-42/2009 respectively. P.S. would like to thank CSIR, India for assistance under SRF Grant 09/045(0736)/2008-EMR-I. This work was initiated during the $X I^{\text {th }}$ Workshop on High Energy Physics Phenomenology held at PRL, Ahmedabad. R.G. and P.S. would like to thank the organisers of the Workshop and the coordinators of the LHC Physics Working Group for their hospitality and cooperation. 


\section{Appendix}

We present here the squared matrix elements for the process $q \bar{q} \rightarrow t \bar{t}$, where, in the subprocess center of mass frame, $t$ has a velocity $\beta$ and subtends an angle $\theta$ with $q$. The final state quarks carry polarizations $\lambda_{t}$ and $\lambda_{\bar{t}}$ respectively. These can then be used to define the requisite density matrices. For each case, one can write

$$
|\mathcal{M}|^{2}=\left|\mathcal{M}_{S M}\right|^{2}+|\mathcal{M}|_{N P}^{2}
$$

where the first part on the r.h.s. represents the SM contribution while the second represents the additional contribution due to the new physics (including the interference with the SM amplitude, and hence not positive definite). For the SM piece, we have,

$$
\left|\mathcal{M}_{S M}\right|^{2}=\frac{g_{s}^{4}}{18}\left[\left(1-\lambda_{t} \lambda_{\bar{t}}\right)\left(1+c_{\theta}^{2}\right)+\left(1+\lambda_{t} \lambda_{\bar{t}}\right)\left(1-\beta^{2}\right)\left(1-c_{\theta}^{2}\right)\right]
$$

where $c_{\theta} \equiv \cos \theta$ and $s_{\theta} \equiv \sin \theta$.

In expressing the $|\mathcal{M}|_{N P}^{2}$ for the respective cases, it is useful to introduce the notation

$$
T \equiv 1-\beta c_{\theta}=\frac{2}{s}\left(m_{t}^{2}-t\right) \quad U \equiv 1+\beta c_{\theta}=\frac{2}{s}\left(m_{t}^{2}-u\right)
$$

\section{$\underline{\text { Triplet Diquark Exchange }}$}

With $y^{2}=y_{S}^{2}+y_{P}^{2}$, we have

$$
\begin{aligned}
|\mathcal{M}|_{N P}^{2}= & \frac{s^{2}}{192\left(u-M_{\phi}^{2}\right)^{2}}\left[y^{4} U^{2}-4 y_{S}^{2} y_{P}^{2} \lambda_{t} \lambda_{\bar{t}}\left(\beta+c_{\theta}\right)^{2}+2 y_{S} y_{P} y^{2}\left(\lambda_{t}-\lambda_{\bar{t}}\right) U\left(\beta+c_{\theta}\right)\right] \\
+ & \frac{g_{s}^{2} s}{72\left(u-M_{\phi}^{2}\right)}\left[y^{2}\left(2-\beta^{2} c_{\theta}^{2}\right)\right. \\
& \left.\quad-\lambda_{t} \lambda_{\bar{t}} y^{2}\left(2 c_{\theta}^{2}+\beta^{2} s_{\theta}^{2}\right)+2 y_{S} y_{P}\left(\lambda_{t}-\lambda_{\bar{t}}\right)\left(\beta+2 c_{\theta}+\beta c_{\theta}^{2}\right)\right] .
\end{aligned}
$$

For the sextet diquark, only the color factors would change [14]. 


\section{$\underline{Z^{\prime} \text { Exchange }}$}

Defining $x \equiv m_{t}^{2} / 2 m_{Z^{\prime}}^{2}$, we have

$$
\begin{aligned}
|\mathcal{M}|_{N P}^{2}=\frac{g_{X}^{4} s^{2}}{16\left(t-M_{Z^{\prime}}^{2}\right)^{2}} & {\left[\left\{U^{2}+2 x\left(1-\beta^{2}\right)+x^{2} T^{2}\right\}\right.} \\
- & \lambda_{t} \lambda_{\bar{t}}\left\{\left(\beta+c_{\theta}\right)^{2}+2 x\left(1-\beta^{2}\right) c_{\theta}^{2}+x^{2}\left(\beta-c_{\theta}\right)^{2}\right\} \\
+ & \left.\left(\lambda_{t}-\lambda_{\bar{t}}\right)\left\{\left(\beta+c_{\theta}\right) U+2 x\left(1-\beta^{2}\right) c_{\theta}-x^{2}\left(\beta-c_{\theta}\right) T\right\}\right] \\
-\frac{g_{s}^{2} g_{X}^{2} s}{18\left(t-M_{Z^{\prime}}^{2}\right)} & {\left[\left\{U^{2}+1-\beta^{2}+x\left(T^{2}+1-\beta^{2}\right)\right\}\right.} \\
- & \lambda_{t} \lambda_{\bar{t}}\left\{(1+x)\left(2 c_{\theta}^{2}+\beta^{2} s_{\theta}^{2}\right)+2(1-x) \beta c_{\theta}\right\} \\
+ & \left.\left(\lambda_{t}-\lambda_{\bar{t}}\right)\left\{\beta(1-x)\left(1+c_{\theta}^{2}\right)+2(1+x) c_{\theta}\right\}\right] .
\end{aligned}
$$

\section{$\underline{\text { Flavor Non-Universal Axigluon Exchange }}$}

Defining combinations of couplings as

$$
\begin{array}{rlrl}
\mathcal{S}_{\mathcal{Q}} & \equiv\left(g_{V}^{q}\right)^{2}+\left(g_{A}^{q}\right)^{2} & \mathcal{P}_{\mathcal{Q}} \equiv g_{V}^{q} g_{A}^{q} & \mathcal{P}_{\mathcal{V}} \equiv g_{V}^{q} g_{V}^{t} \\
\mathcal{S}_{\mathcal{T}}^{ \pm} \equiv\left(g_{V}^{t}\right)^{2} \pm\left(g_{A}^{t}\right)^{2} & \mathcal{P}_{\mathcal{T}} \equiv g_{V}^{t} g_{A}^{t} & \mathcal{P}_{\mathcal{A}} \equiv g_{A}^{q} g_{A}^{t}
\end{array}
$$

we have

$$
|\mathcal{M}|_{N P}^{2}=\frac{s \mathcal{J}_{A}}{18\left[\left(s-M_{A}^{2}\right)^{2}+\Gamma_{A}^{2} M_{A}^{2}\right]}
$$

where

$$
\begin{gathered}
\mathcal{J}_{A}=s\left[\mathcal{S}_{\mathcal{Q}} \mathcal{S}_{\mathcal{T}}^{+}\left(1+\beta^{2} c_{\theta}^{2}\right)+\mathcal{S}_{\mathcal{Q}} \mathcal{S}_{\mathcal{T}}^{-}\left(1-\beta^{2}\right)+8 \mathcal{P}_{\mathcal{Q}} \mathcal{P}_{\mathcal{T}} \beta c_{\theta}\right. \\
-\lambda_{t} \lambda_{\bar{t}}\left\{\mathcal{S}_{\mathcal{Q}} \mathcal{S}_{\mathcal{T}}^{+}\left(\beta^{2}+c_{\theta}^{2}\right)+\mathcal{S}_{\mathcal{Q}} \mathcal{S}_{\mathcal{T}}^{-}\left(1-\beta^{2}\right) c_{\theta}^{2}+8 \mathcal{P}_{\mathcal{Q}} \mathcal{P}_{\mathcal{T}} \beta c_{\theta}\right\} \\
\left.+\left(\lambda_{t}-\lambda_{\bar{t}}\right)\left\{2 \mathcal{S}_{\mathcal{Q}} \mathcal{P}_{\mathcal{T}} \beta\left(1+c_{\theta}^{2}\right)+4 \mathcal{P}_{\mathcal{Q}}\left(\left(g_{V}^{t}\right)^{2}+\beta^{2}\left(g_{A}^{t}\right)^{2}\right) c_{\theta}\right\}\right] \\
+g_{s}^{2}\left(s-M_{A}^{2}\right)\left[\left\{4 \mathcal{P}_{\mathcal{A}} \beta c_{\theta}+2 \mathcal{P}_{\mathcal{V}}\left(2-\beta^{2} s_{\theta}^{2}\right)\right\}\right. \\
-\lambda_{t} \lambda_{\bar{t}}\left\{4 \mathcal{P}_{\mathcal{A}} \beta c_{\theta}+2 \mathcal{P}_{\mathcal{V}}\left(2 c_{\theta}^{2}+\beta^{2} s_{\theta}^{2}\right)\right\} \\
\left.+\left(\lambda_{t}-\lambda_{\bar{t}}\right)\left\{4 g_{A}^{q} g_{V}^{t} c_{\theta}+2 g_{V}^{q} g_{A}^{t} \beta\left(1+c_{\theta}^{2}\right)\right\}\right]
\end{gathered}
$$

For brevity's sake, we are not presenting the SM expressions for $g g \rightarrow t \bar{t}$. 


\section{References}

[1] K. Nakamura et al. [Particle Data Group], J. Phys. G37, 075021 (2010).

[2] T. Aaltonen et al. [CDF Collaboration], Phys. Rev. Lett. 101, 202001 (2008) arXiv:0806.2472 [hep-ex]].

[3] V. M. Abazov et al. [D0 Collaboration], Phys. Rev. Lett. 100, 142002 (2008) arXiv:0712.0851 [hep-ex]].

[4] CDF Conference Note 9724, http://www-cdf.fnal.gov/physics/new/top/public_tprop.html

[5] CDF Conference Note 10185, http://www-cdf.fnal.gov/physics/new/top/public_tprop.html

[6] D0 Conference Note 6062, http://www-d0.fnal.gov/Run2Physics/WWW/results/prelim/TOP/T90/

[7] V. M. Abazov et al. [D0 Collaboration], Phys. Rev. D82 (2010) 032001 arXiv:1005.2757 $[$ hep-ex]].

[8] For a review, see for example,

W. Bernreuther, J. Phys. G35, 083001 (2008) arXiv:0805.1333 [hep-ph]];

T. Han, Int. J. Mod. Phys. A23, 4107 (2008) [arXiv:0804.3178 [hep-ph]].

[9] J. H. Kuhn and G. Rodrigo, Phys. Rev. Lett. 81, 49 (1998) arXiv:hep-ph/9802268]; Phys. Rev. D 59, 054017 (1999) [arXiv:hep-ph/9807420].

[10] M. T. Bowen, S. D. Ellis and D. Rainwater, Phys. Rev. D73, 014008 (2006) arXiv:hep-ph/0509267;

L. G. Almeida, G. F. Sterman and W. Vogelsang, Phys. Rev. D78, 014008 (2008) arXiv:0805.1885 [hep-ph]].

[11] L. M. Sehgal and M. Wanninger, Phys. Lett. B200, 211 (1988).

[12] D. Choudhury, R. M. Godbole, R. K. Singh and K. Wagh, Phys. Lett. B 657, 69 (2007) arXiv:0705.1499 [hep-ph]].

[13] S. Jung, H. Murayama, A. Pierce and J. D. Wells, Phys. Rev. D 81, 015004 (2010) arXiv:0907.4112 [hep-ph]].

[14] J. Shu, T. M. P. Tait and K. Wang, Phys. Rev. D 81, 034012 (2010) arXiv:0911.3237 [hep-ph]].

[15] P. H. Frampton, J. Shu and K. Wang, Phys. Lett. B 683, 294 (2010) arXiv:0911.2955 [hep-ph]].

[16] C. H. Chen, G. Cvetic and C. S. Kim, arXiv:1009.4165 [hep-ph];

Y. K. Wang, B. Xiao and S. H. Zhu, arXiv:1008.2685 [hep-ph];

B. Xiao, Y. K. Wang and S. H. Zhu, Phys. Rev. D 82, 034026 (2010) arXiv:1006.2510 [hep-ph]];

Q. H. Cao, D. McKeen, J. L. Rosner, G. Shaughnessy and C. E. M. Wagner, Phys. Rev. 
D 81, 114004 (2010) [arXiv:1003.3461 [hep-ph]];

V. Barger, W. Y. Keung and C. T. Yu, Phys. Rev. D 81, 113009 (2010) arXiv:1002.1048 [hep-ph]];

J. Cao, Z. Heng, L. Wu and J. M. Yang, Phys. Rev. D 81, 014016 (2010) arXiv:0912.1447 [hep-ph]];

A. Arhrib, R. Benbrik and C. H. Chen, Phys. Rev. D 82, 034034 (2010) arXiv:0911.4875 [hep-ph]];

K. Cheung, W. Y. Keung and T. C. Yuan, Phys. Lett. B 682, 287 (2009) arXiv:0908.2589 [hep-ph]];

A. Djouadi, G. Moreau, F. Richard and R. K. Singh, Phys. Rev. D 82, 071702 (2010) arXiv:0906.0604 [hep-ph]];

C. Degrande, J. M. Gerard, C. Grojean, F. Maltoni and G. Servant, arXiv:1010.6304 [hep-ph];

M. V. Martynov and A. D. Smirnov, arXiv:1010.5649 [hep-ph];

I. Dorsner, S. Fajfer, J. F. Kamenik and N. Kosnik, Phys. Rev. D 81, 055009 (2010) arXiv:0912.0972 [hep-ph]];

G. Burdman, L. de Lima and R. D. Matheus, arXiv:1011.6380 [hep-ph];

E. Alvarez, L. Da Rold and A. Szynkman, arXiv:1011.6557 [hep-ph];

J. A. Aguilar-Saavedra, Nucl. Phys. B 843, 638 (2011) [arXiv:1008.3562 [hep-ph]].

[17] R. S. Chivukula, E. H. Simmons and C. P. Yuan, Phys. Rev. D 82, 094009 (2010) arXiv:1007.0260 [hep-ph]].

[18] D. W. Jung, P. Ko, J. S. Lee and S. h. Nam, Phys. Lett. B 691, 238 (2010) arXiv:0912.1105 [hep-ph]].

[19] K. I. Hikasa, J. M. Yang, B. L. Young, Phys. Rev. D60, 114041 (1999), hep-ph/9908231.

[20] E. Boos, H. U. Martyn, G. A. Moortgat-Pick, M. Sachwitz, A. Sherstnev and P. M. Zerwas, Eur. Phys. J. C 30, 395 (2003) arXiv:hep-ph/0303110;

T. Gajdosik, R. M. Godbole and S. Kraml, JHEP 0409, 051 (2004) arXiv:hep-ph/0405167;

M. Perelstein and A. Weiler, JHEP 0903, 141 (2009) arXiv:0811.1024 [hep-ph]];

M. M. Nojiri and M. Takeuchi, JHEP 0810, 025 (2008) [arXiv:0802.4142 [hep-ph]];

M. Arai, K. Huitu, S. K. Rai and K. Rao, JHEP 1008, 082 (2010) arXiv:1003.4708 [hep-ph]];

K. Huitu, S. K. Rai, K. Rao, S. D. Rindani and P. Sharma, arXiv:1012.0527 [hep-ph].

[21] K. Agashe, A. Belyaev, T. Krupovnickas, G. Perez and J. Virzi, Phys. Rev. D 77, 015003 (2008) arXiv:hep-ph/0612015.

[22] P. S. Bhupal Dev, A. Djouadi, R. M. Godbole, M. M. Muhlleitner and S. D. Rindani, Phys. Rev. Lett. 100, 051801 (2008) arXiv:0707.2878 [hep-ph]].

[23] R. M. Godbole, K. Rao, S. D. Rindani and R. K. Singh, JHEP 1011, 144 (2010) arXiv:1010.1458 [hep-ph]]; AIP Conf. Proc. 1200, 682 (2010) [arXiv:0911.3622 [hepph]]; B. C. Allanach et al., arXiv:hep-ph/0602198.

[24] R. M. Godbole, S. D. Rindani and R. K. Singh, JHEP 0612, 021 (2006) arXiv:hep-ph/0605100. 
[25] J. Shelton, Phys. Rev. D 79, 014032 (2009) arXiv:0811.0569 [hep-ph]];

D. Krohn, J. Shelton, L. T. Wang, JHEP 1007 (2010) 041. [arXiv:0909.3855 [hep-ph]].

[26] M. Jezabek and J. H. Kuhn, Nucl. Phys. B 320, 20 (1989);

A. Czarnecki, M. Jezabek and J. H. Kuhn, Nucl. Phys. B 351, 70 (1991);

A. Brandenburg, Z. G. Si and P. Uwer, Phys. Lett. B 539, 235 (2002) arXiv:hep-ph/0205023].

[27] B. Grzadkowski and Z. Hioki, Phys. Lett. B 476, 87 (2000) arXiv:hep-ph/9911505]; Phys. Lett. B 557, 55 (2003) arXiv:hep-ph/0208079]; Phys. Lett. B 529, 82 (2002) arXiv:hep-ph/0112361;

Z. Hioki, arXiv:hep-ph/0210224; arXiv:hep-ph/0104105,

K. Ohkuma, Nucl. Phys. Proc. Suppl. 111, 285 (2002) [arXiv:hep-ph/0202126].

[28] S. D. Rindani, Pramana 54, 791 (2000) arXiv:hep-ph/0002006].

[29] R. M. Godbole, S. D. Rindani and R. K. Singh, Phys. Rev. D 67, 095009 (2003) [Erratum-ibid. D 71, 039902 (2005)] [arXiv:hep-ph/0211136].

[30] CERN Notes, http://cdsweb.cern.ch/record/814352; http://cdsweb.cern.ch/record/973111.

[31] CDF Conference Note 10211, http://www-cdf.fnal.gov/physics/new/top/confNotes/

[32] D0 Conference Note 5950, http://www-d0.fnal.gov/Run2Physics/WWW/results/prelim/TOP/T84/

[33] J. L. Hewett and T. G. Rizzo, Phys. Rept. 183, 193 (1989).

[34] P. H. Frampton and S. L. Glashow, Phys. Lett. B 190, 157 (1987); Phys. Rev. Lett. 58, 2168 (1987).

[35] T. Aaltonen et al. [CDF Collaboration], Phys. Rev. D 79, 112002 (2009) arXiv:0812.4036 [hep-ex]].

[36] O. Antunano, J. H. Kuhn and G. Rodrigo, Phys. Rev. D 77, 014003 (2008) arXiv:0709.1652 [hep-ph]].

[37] T. Aaltonen et al. [CDF Collaboration], Phys. Lett. B 691, 183 (2010) arXiv:0911.3112 [hep-ex]].

[38] D. Choudhury, M. Datta and M. Maity, Phys. Rev. D 73, 055013 (2006) arXiv:hep-ph/0508009.

[39] T. Aaltonen et al. [CDF Collaboration], Phys. Rev. Lett. 102, 222003 (2009) arXiv:0903.2850 [hep-ex]].

[40] J. Pumplin, D. R. Stump, J. Huston, H. L. Lai, P. M. Nadolsky and W. K. Tung, JHEP 0207, 012 (2002) arXiv:hep-ph/0201195].

[41] CDF Conference Note 9913, http://www-cdf.fnal.gov/physics/new/top/public_xsection.html 
[42] M. Cacciari, S. Frixione, M. L. Mangano, P. Nason and G. Ridolfi, JHEP 0809, 127 (2008) [arXiv:0804.2800 [hep-ph]];

See also,

S. Moch and P. Uwer, Phys. Rev. D 78, 034003 (2008) arXiv:0804.1476 [hep-ph]];

N. Kidonakis and R. Vogt, Phys. Rev. D 78, 074005 (2008) [arXiv:0805.3844 [hep-ph]].

[43] V. Khachatryan et al. [CMS Collaboration], arXiv:1010.5994 [hep-ex].

[44] ATLAS Collaboration, arXiv:1012.1792 [hep-ex].

[45] G. Bhattacharyya, D. Choudhury and K. Sridhar, Phys. Lett. B 355, 193 (1995) arXiv:hep-ph/9504314.

[46] J. Cao, L. Wu and J. M. Yang, arXiv:1011.5564 [hep-ph].

[47] D. W. Jung, P. Ko and J. S. Lee, arXiv:1011.5976 [hep-ph].

[48] T. Aaltonen et al. [The CDF Collaboration], arXiv:1101.0034 [hep-ex]. 